\title{
Uncertainty in the prediction of the thermophysical behavior of new halogenated working fluids
}

Mondejar, Maria E.; Frutiger, Jérôme; Cignitti, Stefano; Abildskov, Jens; Sin, Gürkan; Woodley, John M.; Haglind, Fredrik

Published in:

Fluid Phase Equilibria

Link to article, DOI:

10.1016/j.fluid.2018.12.020

Publication date:

2019

Document Version

Early version, also known as pre-print

Link back to DTU Orbit

Citation (APA):

Mondejar, M. E., Frutiger, J., Cignitti, S., Abildskov, J., Sin, G., Woodley, J. M., \& Haglind, F. (2019). Uncertainty in the prediction of the thermophysical behavior of new halogenated working fluids. Fluid Phase Equilibria, 485, 220-233. https://doi.org/10.1016/j.fluid.2018.12.020

\section{General rights}

Copyright and moral rights for the publications made accessible in the public portal are retained by the authors and/or other copyright owners and it is a condition of accessing publications that users recognise and abide by the legal requirements associated with these rights.

- Users may download and print one copy of any publication from the public portal for the purpose of private study or research.

- You may not further distribute the material or use it for any profit-making activity or commercial gain

- You may freely distribute the URL identifying the publication in the public portal 


\title{
Uncertainty in the prediction of the thermophysical behavior of new halogenated working fluids
}

\author{
Maria E. Mondejar ${ }^{a, *}$, Jérôme Frutiger ${ }^{b}$, Stefano Cignitti ${ }^{\mathrm{b}}$, Jens Abildskov ${ }^{\mathrm{b}}$, \\ Gürkan Sin ${ }^{\mathrm{b}}$, John M. Woodley ${ }^{\mathrm{b}}$, Fredrik Haglind ${ }^{\mathrm{a}}$, \\ ${ }^{a}$ Department of Mechanical Engineering, Technical University of Denmark \\ Building 403, 2800 Kongens Lyngby, Denmark \\ ${ }^{b}$ Department of Chemical and Biochemical Engineering, Technical University of Denmark \\ Building 227, 2800 Kongens Lyngby, Denmark
}

\begin{abstract}
Halogenated olefins can be an attractive alternative to working fluids that are under consideration for phase-out. In order to evaluate the potential of halogenated olefins as working fluids for organic Rankine cycle units with a realistic perspective it is necessary to predict both their primary thermophysical properties and their associated uncertainties. In this work we evaluated the uncertainty of two predictive models, based on the classical group contribution approach and on neural networks, for the critical temperature, critical pressure, acentric factor, and ideal gas heat capacity of halogenated substances. Based on this, guidelines for the most convenient model depending on the property and the fluid molecular structure were provided. The non-linear propagation of the uncertainty through the Peng-Robinson equation of state for the simulation of an organic Rankine cycle unit were also studied. The results suggest that the uncertainty of the predicted properties is highly dependent on the molecular structure for the classical model. The propagation of uncertainties in the simulation of an organic Rankine cycle unit also depends on the saturation properties of the fluid.

Keywords: uncertainty propagation; thermophysical properties; halogenated working fluids; Peng-Robinson equation of state; Monte Carlo simulation.
\end{abstract}

*Corresponding author

Email address: maemmo@mek.dtu.dk (Maria E. Mondejar) 


\begin{tabular}{|c|c|c|c|}
\hline \multicolumn{4}{|c|}{ Nomenclature } \\
\hline$c_{p, 0}$ & $\begin{array}{l}\text { molar ideal gas heat capacity, } \\
\mathrm{Jmol}^{-1} \mathrm{~K}^{-1}\end{array}$ & ORC & organic Rankie cycle \\
\hline$T$ & temperature, $\mathrm{K}$ & PR Fos & Peno-Bobinson eguation of state \\
\hline$p$ & pressure, bar & 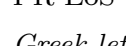 & 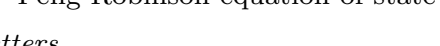 \\
\hline $\mathrm{h}$ & molar enthalpy, $\mathrm{Jmol}^{-1}$ & Greek let & tters \\
\hline Abbre & ations & $\omega$ & acentric factor \\
\hline ANN & artificial neural network model & $\rho$ & molar density, molm ${ }^{-3}$ \\
\hline cEoS & cubic equation of state & $\sigma$ & standard deviation \\
\hline EoS & equation of state & Subscrip & \\
\hline GCM & classical group contribution model & $\mathrm{b}$ & boiling \\
\hline GWP & $\begin{array}{l}\text { global warming potential over } 100 \text { years } \\
\text { relative to } \mathrm{CO}_{2}\end{array}$ & $\mathrm{c}$ & critical \\
\hline $\mathrm{HFC}$ & hydrofluorocarbons & $\exp$ & experimental \\
\hline $\mathrm{HFO}$ & hydrofluoroolefins & liq & saturated liquid \\
\hline ODP & $\begin{array}{l}\text { ozone depletion potential relative to } \\
\text { CFC- } 11\end{array}$ & $\begin{array}{l}\text { pred } \\
\text { vap }\end{array}$ & $\begin{array}{l}\text { predicted } \\
\text { saturated vapor }\end{array}$ \\
\hline
\end{tabular}

\section{Introduction}

In the last few years environmental regulations have been a major driving force for the industry to seek novel working fluids, as many of the conventional fluids in use today will be soon phased out. Although the Montreal Protocol in 1989 [1] and the Kyoto Protocol in 1997 [2] already imposed restrictions on the use of some refrigerants, other regulations of regional coverage, such as the Regulation on Fluorinated Substances of the European Parliament (F-gas regulation)[3], impose now additional requirements and limitations. As a matter of fact, on $15^{\text {th }}$ October 2016 a total of 197 countries agreed on the amendment of the F-gas regulation to the Montreal Protocol, thus extending its phase-out schedule for hydrofluorocarbons (HFCs) within the United Nations. This agreement will have a significant impact on the existing refrigeration units and organic Rankine cycle (ORC) power systems utilizing HFCs for their operation, increasing the efforts for the search of alternative fluids. However, the design of new working fluids poses a great challenge, and finding an optimum solution for a given application often faces tradeoffs between cycle performance, and environmental and safety criteria.

Halogenated olefins (also called hydrofluoroolefins (HFOs)) offer good en- 
vironmental characteristics (i.e., low or zero ozone depletion potential (ODP) and low global warming potential (GWP)), which is why their potential as working fluids for ORC units and refrigeration cycles is proposed. With the aim of evaluating the prospects of new halogenated olefins, in our previous work [4] we developed two alternative models based on the group contribution approach, for the prediction of the critical temperature $T_{\mathrm{c}}$, critical pressure $p_{c}$, acentric factor $\omega$, normal boiling temperature $T_{b}$, and ideal gas heat capacity $c_{\mathrm{p}, 0}$ of organic compounds containing fluorine and/or chlorine, based on an extended database of properties. The models were based on a classical and a neural-network-based group contribution approaches. It was observed that the two developed models improved the predictions for all properties with respect to other group contribution methods and molecular structure models available in the literature. Moreover, it was observed that the model based on a neural network approach yielded lower deviations from the experimental data than the one following the classical approach of the group contribution method for all properties but for the acentric factor.

In order to apply these predictive models for the comprehensive evaluation of the potential of new halogenated working fluids in ORC power systems and refrigeration systems it is necessary to evaluate and compare the uncertainty of their predictions, and analyze how the uncertainty propagates when the predicted values are used as inputs in equations of state (EoS) to simulate industrial processes. In this work, we distinguish between the terms uncertainty and deviation. Uncertainty is considered here to be the range of statistically possible outcomes of a property model, for example expressed with $95 \%$ confidence, or the standard deviation of a (normal) distribution representing these outcomes [5]. Deviation refers, here, to the relative or absolute difference between the predicted value of a model and the value of the corresponding experimental measurement of the property.

The influence of the uncertainty of predicted fluid properties on the study of their thermophysical behavior in different processes has been previously analyzed by other authors. For instance, Whiting [6] raised the importance of analyzing the impact of the uncertainty of physical property data and predictive models on the simulation results of several industrial separation processes. Hajipour and Satyro [7, 8] presented the propagation of the uncertainty of the critical properties and acentric factor of large chain hydrocarbons through the Peng-Robinson EoS (PR-EoS) and developed a correlation for their prediction based on the experimental uncertainty of the available data. Bjorner et al. [9] analyzed the propagation of the uncertainty in 
the parameters of cubic plus association equations of state. More recently, Shacham et al. [10] considered the experimental uncertainty of the physical properties of fluids to account for the worst case scenario of the design of a heat exchanger and tubular reactor. Also, Hukkerikar et al. [11] analyzed the sensitivity of process design to the uncertainties in a set of thermophysical properties for the case study of an extractive distillation process. In the domain of working fluids for ORC power systems, the propagation of property uncertainties to the model output has been suggested as a promising tool to compare the uncertainties in property prediction methods on the cycle level [12]. In this regard, Frutiger et al. [13] utilized a Monte Carlo method to propagate the uncertainty of the fluid-specific equations of state parameters to the cycle model output.

Nevertheless, a common problem when analyzing the uncertainty propagation of fluid properties in process design is the lack of uncertainty values for the predicted properties. Although the prediction uncertainty of several classical group contribution methods, such as Joback and Reid [14] and Marrero and Gani [15], is unknown, it is expected that longer repeating chains of molecular groups will have higher deviations, whereas shorter chained molecules with varying groups will have lower deviations. In order to overcome the lack of uncertainty values from these predictive models, Hukkerikar et al. [16] presented a method based on the Marrero and Gani [15] model with new contributions, additional properties, and reported uncertainties. However, as many groups that describe halogenated fluids are missing, the uncertainties are expected to be high for them if their contributions were estimated with the connectivity index method [17], which adds uncertainty to the prediction. Moreover, Frutiger et al. [18] presented recently a methodology for the uncertainty estimation of group contribution based property models, which provides every predicted data point along with its respective uncertainty range.

The aforementioned publications deal with the analysis of the impact of experimental or predicted property uncertainty on the design or simulation of different processes, and how these uncertainties are determined in the case of developed predictive models. In this work we aim to evaluate how the nature of the predictive model or the fluid molecular structure influence the expected uncertainty of predicted properties for halogenated organic fluids. Then the impact of the selection of the predictive models on the overall uncertainty of the results in the study of new halogenated substances in power cycle systems is analyzed. The necessary tools for the estimation of the uncertainty of new 
halogenated fluids are provided enabling researchers in the field to assess the propagation of the uncertainty of the predictions in their simulation models. This work presents a number of novel contributions. First, an evaluation of the uncertainty of prediction models for the thermophysical properties of halogenated substances is provided. Second, the uncertainty of the predicted values obtained from models based on different approaches (group contribution methods and artificial neural networks) are compared, giving a first insight into what could be the advantages or disadvantages of using these models for different fluids and properties, in terms of the uncertainty of their predictions. Third, based on the results, a series of guidelines on the convenience of using specific models depending on the deviations of their predictions, the uncertainty, and the fluid molecular structure, are provided.

First, the paper introduces, in Section 2, the methods followed for the estimation of the uncertainty of the predicted properties, the propagation of the uncertainty through the PR-EoS, and the estimation of the uncertainty propagation on the case study of an ORC unit. Section 3 presents and compares the results obtained for each of the predictive methods and properties. Finally, the results are discussed in Section 4, including an analysis on the uncertainty improvement based on the availability of experimental data and a comparison with the expected uncertainties from other models. The main conclusions of this work are summarized in Section 6. An example of estimation of the uncertainty and additional material is provided in the Supporting Information (see section 5).

\section{Methods}

\subsection{Prediction of thermophysical properties}

The easiest method to predict the thermophysical behavior of a certain fluid is by using a cubic equation of state, where only the critical temperature $T_{\mathrm{c}}$, critical pressure $p_{\mathrm{c}}$, the acentric factor $\omega$, and the ideal gas heat capacity $c_{\mathrm{p}, 0}$ are needed. The experimental values of these parameters can be easily accessed for a large number of substances through databases, or they can also be predicted if no experimental data are available. In this regard, the greatest challenge that arises when attempting to predict the thermophysical behavior of new working fluids is the lack of experimental data of their core properties. As a consequence, the development of models for predicting different properties of new fluids of interest is a topic of high interest for both researchers and industry. 
In our previous work [4] we developed two models, based on a group contribution approach, to predict the critical temperature $T_{\mathrm{c}}$, critical pressure $p_{\mathrm{c}}$, acentric factor $\omega$, and ideal gas heat capacity $c_{\mathrm{p}, 0}$ of halogenated organic substances:

1. The first model (from now on referred to as the GCM) followed the classical approach of the group contribution method, as described by Marrero and Gani [15],

2. The second model (from now on referred to as the ANN) combined the use of artificial neural networks with the group contribution approach.

The results indicated that the two developed methods were able to predict the mentioned properties with lower deviations from the experimental values, than with other available molecular-based methods. Moreover, it was found that the model following the neural network approach (ANN) yielded lower absolute average relative deviations than the model following the classical approach (GCM) for all the studied properties, except for the acentric factor. This success in improving the predictive performance was due to the reduction of the scope of the method to a specific fluid group, the use of an extensive database of experimental data, and the use of neural networks, in the case of the ANN.

However, it was unclear whether the improvement in the prediction capacity of the ANN was accompanied by a reduction in the uncertainty of the prediction, and which of the two methods yielded a lower uncertainty in the prediction of the properties. Moreover, it is important to study how the uncertainty of the predicted values by using both models propagates through the use of cubic equations of state, in order to evaluate if initial differences on the uncertainty values are smoothed in the final simulation results.

\subsection{Estimation of fluid behavior with cubic equations of state}

As mentioned previously, the easiest way to predict the thermophysical behavior of fluids for which limited experimental data are available is by using cubic equations of state $(\mathrm{cEoS})$. A cEoS is an equation of state that provides the pressure as a function of temperature and volume, being roots third order in volume (i.e., there are three real and/or imaginary solutions for the volume for a specific temperature $T$ and pressure $p$ ). The general form of a cEoS is given in Eq. 1 [19].

$$
p=\frac{R T}{v-b}-\frac{a_{c} \alpha\left(T_{R}, \omega\right)}{\left(v+\Delta_{1} b\right)\left(v+\Delta_{2} b\right)}
$$


Here $a_{c}$ and $b$ are the attraction and co-volume parameters, respectively, that are calculated with the critical temperature $T_{\mathrm{c}}$, critical pressure $p_{\mathrm{c}}$, and acentric factor $\omega$ of the fluid, and $\alpha$ (the so-called attraction term) is a function of the temperature, and/or the acentric factor. $\Delta_{1}$ and $\Delta_{2}$ are parameters whose values determine the type of cEoS.

The advantage of the use of $\mathrm{cEoS}$ is that reasonably accurate predictions can be expected if only the critical parameters, the acentric factor, and the ideal gas heat capacity of the fluid are known. Moreover, the computing time required by the calculation subroutines is, in many cases, significantly lower than that required by other equations of state (e.g., the Helmholtz-free energy based EoS, which can offer a more accurate representation at the expense of more complexity and the requirement of fluid-specific parameters). The predictions of cEoS are more accurate in the vapor phase, and when the fluid is non-polar, but are known to fail in the estimation of the compressed liquid volume, and near the critical region. In this context, Brown [20] demonstrated that using $\mathrm{cEoS}$ is a reliable method to study the performance of new refrigerants in vapor compression systems, even in the absence of experimental data for the critical parameters and ideal gas heat capacity. The work showed that, when these parameters are known, relative deviations of less than $5 \%$ can be expected for the predicted properties with respect to those obtained from the Helmholtz-based equations of state of Refprop [21]. Liu et al. [22] observed similar deviations when using the PR-EoS to evaluate the potential of a set of HFO as working fluids of ORC power systems.

Nevertheless, the aforementioned studies only evaluated the predictive performance of the PR-EoS, without considering how the uncertainty of the critical parameters, acentric factor, or ideal gas heat capacity propagated in the predictions of the EoS. In this context, Frutiger et al. [12] recently analyzed the uncertainty propagation of these parameters in the Soave-RedlichKwong EoS, for the case of an ORC unit. Previously, Frutiger et al. [13] used the propagation of the experimental uncertainties of the critical parameters, acentric factor, and ideal gas heat capacity through the PR-EoS for the optimization of the working fluid selection in an ORC unit.

In this work we study the propagation of predicted property uncertainties for halogenated fluids on the PR-EoS (for which the values of $\Delta_{1}$ and $\Delta_{2}$ in Eq. 1 are $\Delta_{1}=1+\sqrt{2}$ and $\Delta_{2}=1-\sqrt{2}$, and the parameters $a_{c}$ and $b$ are defined as $a_{c}=0.45724 R^{2} T_{c}^{2} / p_{c}$ and $\left.b=0.07780 R T_{c} / p_{c}\right)$, since this is the most widely used cubic EoS. 


\subsection{Uncertainty analysis}

In this section the methods used for the evaluation and propagation of the uncertainty are described. First, the availability of the experimental uncertainty in the property database is discussed. For the two considered models (GCM and ANN) the uncertainty of the predicted property values was estimated by using the bootstrap method, and the $95 \%$ confidence interval of the predicted properties is provided. Next, the propagation of these estimated uncertainties through the PR-EoS is explained, and the propagation of uncertainties on a case study of an ORC unit is presented. This method allows the comparison of the uncertainty generated by the GCM and the ANN, both at the level of the prediction and at the level of the cycle simulation. Figure 1 provides an overview of the method.

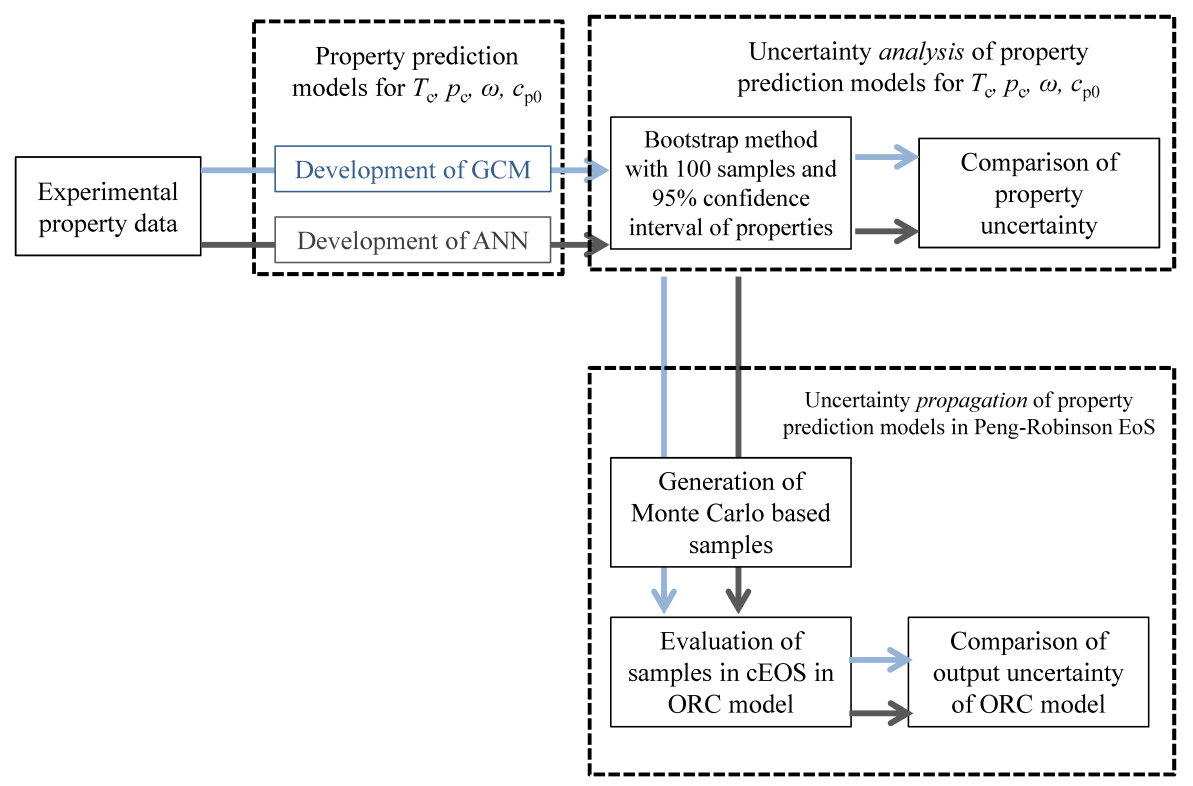

Figure 1: Schematic overview of the method for uncertainty analysis and propagation.

\subsubsection{Uncertainty of experimental data}

In our previous work [4] we collected an extensive dataset containing the experimental critical temperature, critical pressure, normal boiling temperature, acentric factor, and ideal gas heat capacity constants of up to 334 organic fluids containing fluorine and/or chlorine. In this set, only $75 \mathrm{flu}-$ ids included information about their associated measuring uncertainty for 
the critical temperature and critical pressure, and only 14 included information about the uncertainty for the acentric factor (corresponding to those data obtained from the database of the Project 801 of the Design Institute for Physical Property Data (DIPPR ${ }^{\circledR} 801$ ) [23]). Regarding the ideal gas heat capacity, this database reports the constants of the Aly-Lee model for heat capacity [24] for 31 fluids. In this case the fitting errors for each of the constants are reported. This situation represents a drawback for the comprehensive evaluation of the uncertainty of the predictions of the models developed in Ref. [4], since the few experimental uncertainties available in the dataset cannot provide an insight of the overall quality of the data, nor the presence of outliers. Since the impact of including the few available experimental uncertainties in the analysis would be low, it was decided not to include them into the uncertainty propagation analysis. Instead, the experimental uncertainties are later compared with the calculated uncertainties from both models in section 4 .

\subsubsection{Uncertainty of predicted data}

While most of the published models for the prediction of thermophysical properties lack an assessment of the uncertainty of their predictions, this practice is recommended, and should be standardized [25]. When, as in our case, the uncertainty of the experimental data used for the fitting of the predictive models is only partially available, the uncertainty analysis provides the estimated uncertainty of the fitted model, independently of the quality of the experimental data. Thus, the results must be seen as a measure of the quality of the fitting of the model, but should be compared with the available experimental uncertainties to assess the total expected uncertainty of the predicted property value.

The parameters fitted in group contribution methods correspond to the individual contributions of each functional group and, in some cases, function coefficients. In the case of the neural network based GCM, the number of fitted parameters is greater than the number of functional groups, and there is no direct relationship between them. The parameters of the group contribution based models developed in our previous work [4] were obtained by nonlinear least-squares regression of the predicted values of each property (for the GCM) and by the minimization of the mean of squared errors (for the ANN). In this optimization method, the residuals of the predicted values were minimized in order to determine the values of the parameters, as indicated in Eq. 2. 


$$
\theta^{*}=\operatorname{argmin}\left(\sum_{i}\left(y_{i}^{\text {exp }}-y_{i}^{\text {pred }}\right)^{2}\right)=\operatorname{argmin}\left(\sum_{i}\left(\epsilon_{i}\right)^{2}\right)
$$

Here, $\theta^{*}$ is the array of fitted parameters, and $y_{i}^{\exp }$ and $y_{i}^{\text {pred }}$ are the experimental and predicted values of a specific property $y$ for each fluid $i$ in the dataset, and $\epsilon_{i}$ is the calculated error. The datasets used in this work correspond to those used in our previous work [4], which collected experimental data for halogenated substances from the database of the Project 801 of the Design Institute for Physical Property Data (DIPPR 801 [23]) of $T_{\mathrm{c}}$ , $p_{\mathrm{c}}, \omega$, and calculated values of $c_{\mathrm{p}, 0}$ from the Aly and Lee constants [24] at reduced temperatures of $0.6,0.65,0.7,0.75,0.8,0.85$, and 0.9 . For each of the properties, the predicted value $y_{i}^{\text {pred }}$, predicted by the GCM and the ANN, was obtained as indicated in Eqs. 3 and 4, respectively:

$$
\begin{aligned}
& f\left(y_{i}^{\text {pred }}\right)=\sum_{j} N_{j} C_{j}+\sum_{k} M_{k} D_{k} \\
& f\left(y_{i}^{\text {pred }}\right)=f\left(w_{\mathrm{ANN}}, b_{\mathrm{ANN}}, N_{j}, M_{k}\right)
\end{aligned}
$$

Here $f\left(y_{i}^{\text {pred }}\right)$ is a function of the property to be predicted, and $j$, and $k$ refer to the first, and second order groups defined in the GCM. $N_{j}$ and $M_{k}$ are the number of the j-th first order group, and the k-th second order group, respectively, present in the molecule, and $C_{j}$ and $D_{k}$ are the fitted contributions of the GCM to the first and second order groups, respectively. $w_{\mathrm{ANN}}$ and $b_{\mathrm{ANN}}$ refer to the fitted weights and biases of the artificial neural network of the ANN. The fitted neural networks were composed of one or two hidden layers, depending on the property. The number of neurons per layer, transfer function, groups decomposition, and the values of the fitted contributions, weights, and biases for each model and property can be consulted in Ref. [4].

Frutiger et al. [18] compared two different methods (i.e linear error propagation with parameter covariance matrices, and the bootstrap method) to evaluate the uncertainty of values predicted with group contribution models. The authors found that the bootstrap method was a valid alternative to the classical uncertainty analysis based on linear error propagation, especially when the residuals are not normally distributed and/or not independent. In order to check whether the prerequisites required for linear propagation were meet in this work, a one-sample Kolmogorov Smirnov test [26] was used to 
test the distribution of the residuals obtained for both models (GCM and ANN) and for each of the considered properties. The test yielded a positive result for the GCM for all properties and for the ANN for $c_{\mathrm{p}, 0}$, which means that for these cases the residuals do not follow a normal distribution. The result can be observed in Figure 2, where the normal probability plots of the residuals for each predictive model are represented. The linear correlation of the residuals for each predictive model and property was checked against the prediction values and input variables, and was found lower than 0.35 for all the cases except for the ANN for $c_{\mathrm{p}, 0}$ where a high correlation was observed.

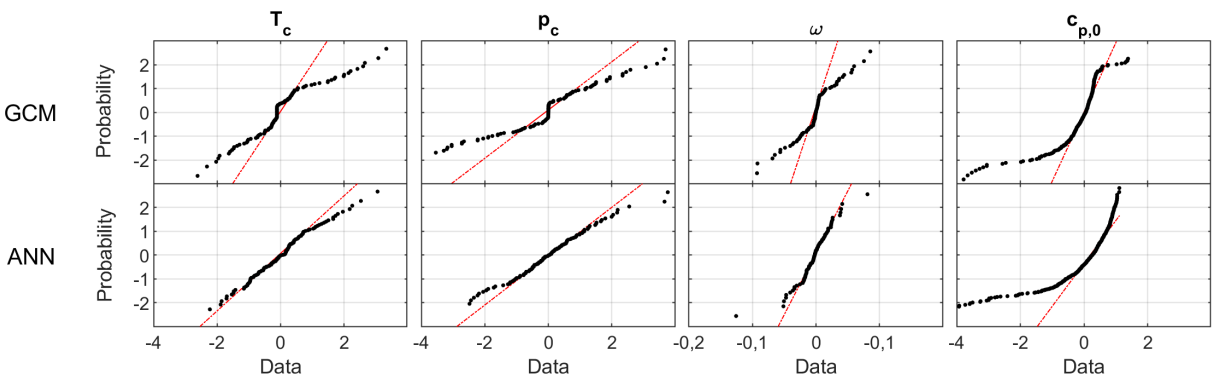

Figure 2: Normal probability plots of the residuals of the predictive models GCM and ANN (in rows) for the properties $T_{\mathrm{c}}, p_{\mathrm{c}}, \omega, c_{\mathrm{p}, 0}$ (in columns).

Because of the non-normal distribution of the residuals $\epsilon_{i}$ in the abovementioned cases, the bootstrap method was selected to evaluate the uncertainty of the predictions in this work. The bootstrap method approximates the distribution of the residuals $\hat{F}$ by assuming that the residuals are uniformly distributed, $\hat{F}=\operatorname{mass} \frac{1}{n}$. With this distribution, a number $n$ of synthetic datasets is generated, as indicated in Eq. 5. In this work, 100 synthetic datasets were generated for each of the studied properties and models (for more information on the choice of the number of samples, please refer to the Supporting Information).

$$
y_{i}^{*}=y_{i}^{\operatorname{pred}}(\theta)+\hat{\epsilon}_{i}, \hat{\epsilon}_{i} \in(\hat{F})
$$

Then, the model parameters $\theta^{*}$ are re-fitted for each of the generated datasets, and new predictions $y_{i}^{\text {pred,* }}$ are obtained with these parameters. This results in a set of parameters and predictions from which the mean and standard deviation can be calculated by using inference statistics, as indicated in Eqs. 6 and 7 
335

$$
\mu_{\theta^{*}}=\frac{1}{n} \sum_{k=1}^{n} \theta^{*}(k)
$$

$$
\sigma_{\theta^{*}}=\sqrt{\frac{1}{n-1} \sum_{k=1}^{n}\left(\theta^{*}(k)-\mu_{\theta^{*}}\right)^{2}}
$$

Percentile inference statistics were also obtained from the resulting parameter estimation matrices from their empirical cumulative probability distributions, to allow the comparison of the uncertainty ranges of the properties predicted from different models.

\subsubsection{Uncertainty prediction of new halogenated working fluids}

While the analysis of uncertainties presented in sections 3.1 and 3.2 concerns the fluids included in the database used for the development of the models presented by Mondejar et al. [4], the main interest of this analysis is to provide a tool for the estimation of the uncertainty for the predictions of the critical temperature, critical pressure, acentric factor, and ideal gas heat capacity of new halogenated working fluids. The method used to predict the uncertainty of the property predictions of new fluids involves a reverse bootstrapping process, so that for each property and model, a number of samples of model parameters is generated based on their mean value and the estimated uncertainties as calculated in Eqs. 6 and 7, and by considering the specific probability distribution function (PDF) obtained from the Bootstrap method for each of the parameters (in order to avoid errors derived from the assumption of normal distribution for non-normal distributed parameters). The property value was obtained for each of the generated parameters and its uncertainty was obtained from the set of calculated values. The code of a function executing this method is provided in the Supporting Information (see Section 5).

\subsubsection{Uncertainty propagation in the Peng-Robinson equation of state}

When predicted values of the critical temperature $T_{\mathrm{c}}$, critical pressure $p_{\mathrm{c}}$, and acentric factor $\omega$ are used as inputs in a cEoS to estimate the thermophysical behavior of a fluid, and the ideal gas heat capacity $c_{\mathrm{p}, 0}$ is used to calculate its enthalpy and entropy, their associated uncertainties are propagated to the properties derived from the EoS. In order to evaluate the uncertainty of the thermophysical properties obtained from the PR-EoS, an 
in-house code of the PR-EoS, developed in Matlab (version R2015a, The Mathworks, Natick, MA) was used.

The method proposed by Frutiger et al. [13] was used for a Monte Carlo based uncertainty propagation. First, a set of 100 samples of $T_{\mathrm{c}}, p_{\mathrm{c}}, \omega$, and $c_{\mathrm{p}, 0}$ was created for the Monte Carlo simulation by using the Latin hypercube sampling method [27] (please refer to the Supporting Information for more information regarding the samples number). All the properties were assumed to follow a normal distribution where the standard deviation was equal to the predicted uncertainty from the model. The correlation between the model input parameters for each property was considered during the sample generation by using the rank-based method for correlation control proposed by Iman and Conover [28]. A graphical representation of the correlation matrices of each property is provided in Figures 3 and 4 of the Supporting Information. Then, a set of thermophysical properties of interest for the study of ORC and refrigeration units (i.e., saturation pressure, saturated densities, and saturated enthalpies) was calculated by using the PR-EoS for selected fluids, and the distribution of the results was analyzed.

\subsubsection{Uncertainty propagation in an organic Rankine cycle unit}

The propagation of the uncertainty of the predicted properties within the simulation of an ORC unit operating with a halogenated olefin as the working fluid was used as a case study to estimate the impact of predicted properties' uncertainties on process simulation. The diagram of the ORC unit model used in this work is shown in Figure 3.

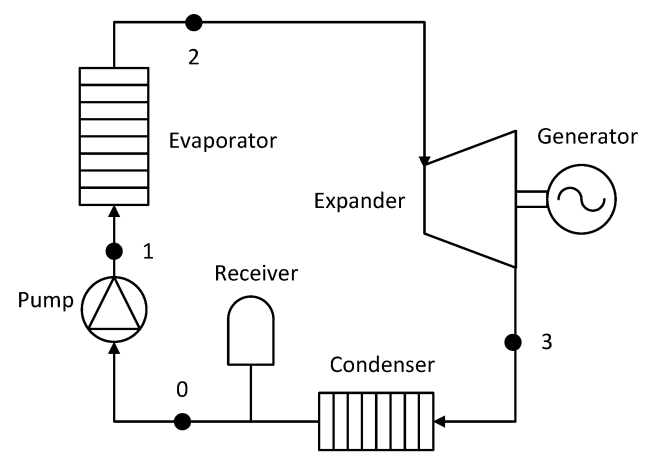

Figure 3: Schematic diagram of an ORC unit.

The saturated liquid exiting the condenser (state 0) is pumped to the 
maximum pressure of the cycle (state 1), and then is evaporated with the energy from the heat source up to saturated or superheated vapor conditions (state 2). Afterwards the fluid flows through the expander (state 3) and enters the condenser to complete the cycle. The model has a standard configuration with no regeneration and without subcooling conditions (i.e., the working fluid at the outlet of the condenser is saturated liquid). The net power output of the unit was obtained as:

$$
W_{\text {net }}=\eta_{\text {gen }}\left(W_{\text {exp }}-W_{\text {pump }}\right)=\eta_{\text {gen }} m_{\mathrm{ORC}}\left(\eta_{\mathrm{t}}\left(h_{2}-h_{3}\right)-\left(h_{1}-h_{0}\right) / \eta_{\mathrm{p}}\right)
$$

where $\eta_{\text {gen }}, \eta_{\mathrm{t}}$, and $\eta_{\mathrm{p}}$ are the efficiencies of the generator, the expander, and the pump, respectively; $m_{\mathrm{ORC}}$ is the working fluid mass flow rate, and $h_{i}$ refers to the specific enthalpy on each of the states of the cycle, marked in Figure 3. The efficiency of the generator, and the isentropic efficiencies of the pump and expander were set to constant values equal to $\eta_{\text {gen }}=0.975$, $\eta_{\mathrm{p}}=0.4$ and $\eta_{\mathrm{t}}=0.7$, respectively. The thermal efficiency of the cycle is obtained as $\eta_{\mathrm{ORC}}=W_{\text {net }} / Q_{\text {in }}$, where $Q_{\text {in }}$ is the heat transferred from the heat source to the working fluid in the evaporator.

For each of the predictive methods (i.e., GCM and ANN) a set of 100 samples of $T_{\mathrm{c}}, p_{\mathrm{c}}, \omega$, and $c_{\mathrm{p}, 0}$ constants was generated by using the Latin hypercube sampling method for the Monte Carlo method. Then, a Monte Carlo simulation of the ORC unit was performed by considering the set of samples, and the distribution of the resulting net power output $W_{\text {net }}$ and thermal efficiency $\eta_{\mathrm{ORC}}$ values was analyzed. The heat source of the ORC unit was assumed to be a hot stream of water at $358.85 \mathrm{~K}$ and the sink was considered as water at $291.55 \mathrm{~K}$, resembling a hypothetical case where an ORC unit recovers the heat from the jacket cooling water of a ship's propulsion Diesel engine, using seawater as a sink. The process simulation parameters of the ORC unit were obtained for the halogenated olefins trans1-chloro-3,3,3-trifluoropropene (R-1233zd(E)) and 2,3,3,3-tetrafluoropropene (R-1234yf), by following the optimization procedure described in the work of Andreasen et al. [29], for the maximization of the net power output. The values of these parameters are given in Table 1. Pressures losses in the unit piping and heat exchangers were neglected for simplicity. 
Table 1: Process simulation parameters of the case study ORC unit, optimized for maximum net power output.

\begin{tabular}{lll}
\hline Parameter & Values & \\
\hline Working fluid & $\mathrm{R}-1233 \mathrm{zd}(\mathrm{E})$ & $\mathrm{R}-1234 \mathrm{yf}$ \\
Source mass flow rate, $\mathrm{kg} \mathrm{s}^{-1}$ & 35.9 & 35.9 \\
Source temperature, K & 358.9 & 358.9 \\
Sink temperature, K & 291.6 & 291.6 \\
Boiler pressure, bar & 3.7 & 16.4 \\
Condensation temperature, K & 307.2 & 307.2 \\
Expander inlet superheat, K & 5.0 & 12.3 \\
Pump inlet subcooling, K & 6.0 & 6.0 \\
\hline
\end{tabular}

\section{Results}

\subsection{Uncertainty analysis of the GCM and the ANN models}

Figure 4 presents, for each of the analyzed properties, the relative deviation of the predicted values of the two considered models, from the experimental values. The error bars show the calculated uncertainty from the procedure described in section 2.3 for the GCM (figures on the left side) and the ANN (figures on the right side). As can be seen, the average relative deviation is slightly lower for all the properties when obtained from the ANN, with a significant decrease in the case of the ideal gas heat capacity. However, no significant difference can be seen in the uncertainty values between the methods, except for the ideal gas heat capacity where the uncertainty appears significantly lower for the ANN. For both methods, the relative uncertainties appear to decrease for higher values of the property (which is correlated with larger molecules), as expected. This is very clear in the case of the prediction of $c_{\mathrm{p}, 0}$ from the GCM, where there are higher uncertainty values at lower heat capacity values.

\subsection{Uncertainty of EoS properties}

\subsubsection{Sensitivity of EoS to input properties}

The uncertainty of the input magnitudes to the PR-EoS (i.e., $T_{\mathrm{c}}, p_{\mathrm{c}}$, $\omega$, and $\left.c_{\mathrm{p}, 0}\right)$ was propagated in a different way for each of them, and for different EoS properties, which means that their impact on the final uncertainty is not homogeneous. Figure 5 presents how an assumed uncertainty of $5 \%$ on each of the input properties is propagated through the PR-EoS for different thermophysical properties of difluoromethane (R-32), at a temperature of $T=300 \mathrm{~K}$. This hypothetical and relatively high value is chosen for 
GCM

a)

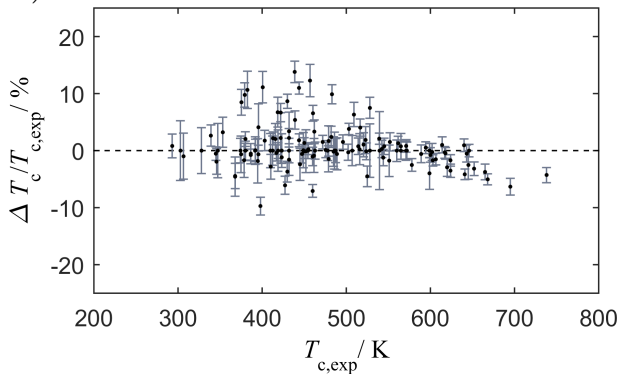

c)

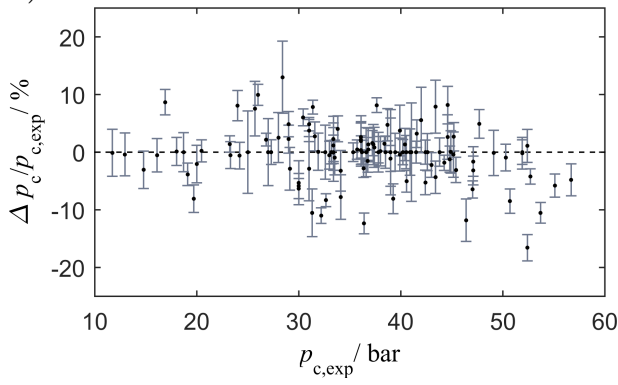

e)

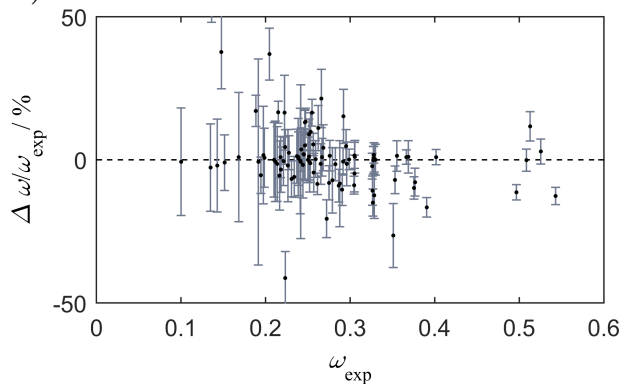

g)

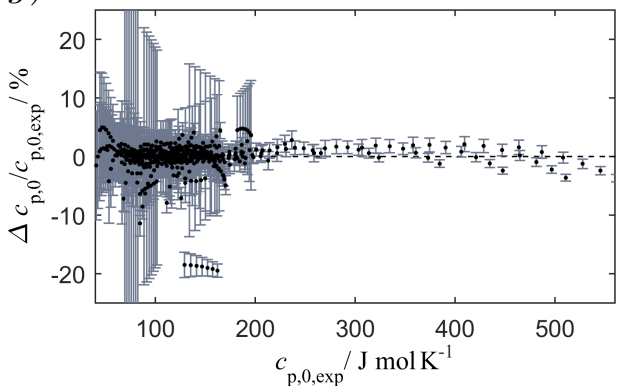

ANN

b)

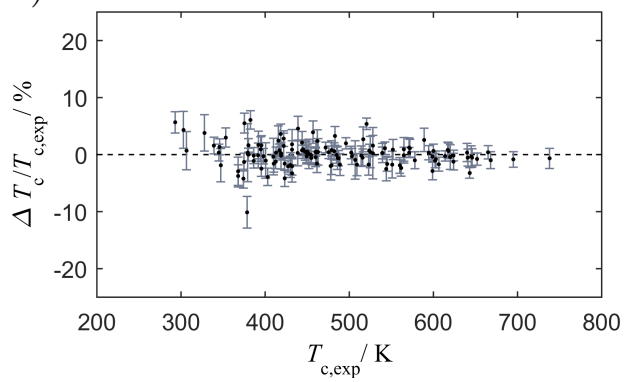

d)

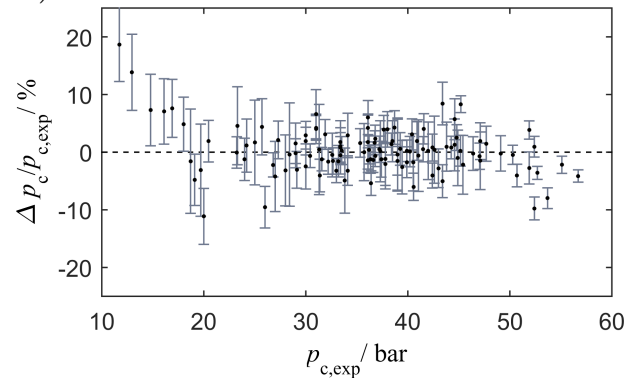

f)

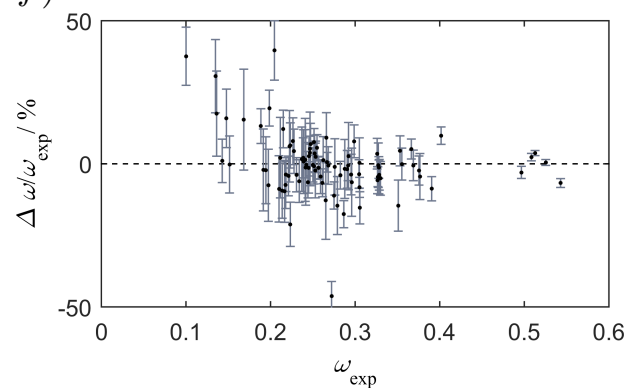

h)

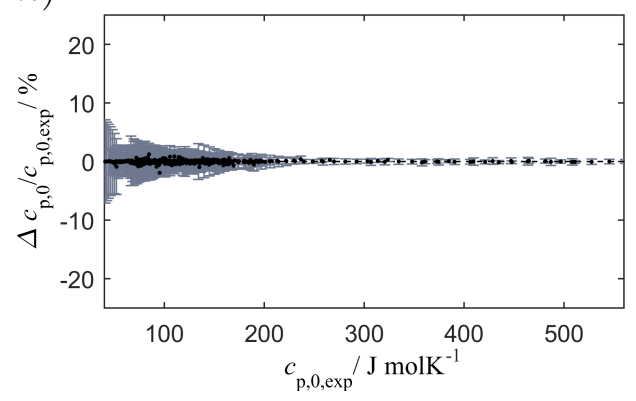

Figure 4: Relative deviations of the predicted values of ( $a$ and $b$ ) the critical temperature, (c and d) critical pressure, (e and f) acentric factor, and ( $g$ and $h$ ) ideal gas heat capacity vs. the database values. Error bars show the uncertainties of the predicted values by using the GCM (figures on the left side) or the ANN (figures on the right side). 
a better readability of the propagation results. For each input property, the bar represents the uncertainty of the calculated property when the indicated input variable has an uncertainty of $5 \%$, and null uncertainty is assumed for the rest of the input properties.

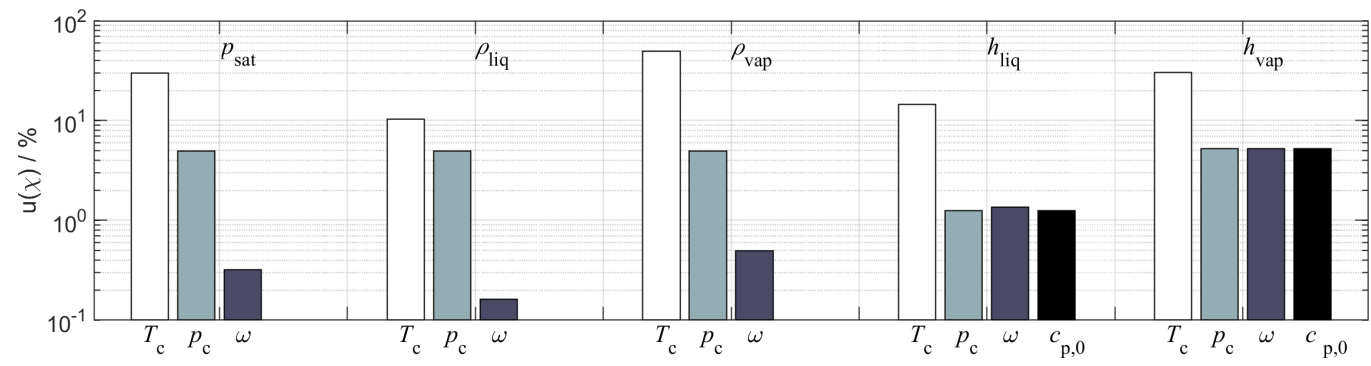

Figure 5: Impact of the uncertainties of the input variables (i.e., $T_{\mathrm{c}}, p_{\mathrm{c}}, \omega$, and $c_{\mathrm{p}, 0}$ ) on the overall uncertainty of $p_{\text {sat }}, \rho_{\text {liq }}, \rho_{\text {vap }} h_{\text {liq }}$, and $h_{\text {vap }}$. For each of the listed properties $\chi$, the bars represent its overall uncertainty $u(\chi)$ when a $5 \%$ uncertainty is considered only on the corresponding input property at the bottom. Note the logarithmic scale on the y-axis. The enthalpy calculations correspond to a saturation temperature of $300 \mathrm{~K}$.

As it can be seen, the uncertainty of the critical temperature affects the overall uncertainty more than any other input property. An uncertainty of $5 \%$ in the critical temperature results in an uncertainty of more than $10 \%$ for the calculated saturated pressure, and saturated vapor density and enthalpy. The uncertainty of the critical pressure has a similar impact on the saturation pressure, and saturated densities, resulting in an overall uncertainty of around $2 \%$, and less in the case of the saturated liquid enthalpies. The uncertainty in the acentric factor has a larger impact on the saturated enthalpies, similar to that of the ideal gas heat capacity, but still remains negligible compared to the impact of the uncertainty in the critical temperature. It is also observed that the impact of the ideal gas heat capacity uncertainty is greater for the saturated vapor enthalpy, which is expected, as the ideal enthalpy is larger than the residual enthalpy in the vapor calculation, whereas in the liquid calculation the residual term is dominant, and is affected by the uncertainties in the critical parameters.

These results indicate that the critical temperature is the key in order to reduce the uncertainty propagated through the PR-EoS, whereas reducing the uncertainty of the other properties will not have a similar impact on the total property uncertainty. 


\subsubsection{Examples of propagation in the PR-EoS}

In order to illustrate how the uncertainty in the input properties is propagated through the use of the PR-EoS, two fluids were selected from the set to represent two cases with large and low estimated uncertainties for the considered properties (based on the values from the GCM). The selected fluids are 2,2-dichloropropane and propyl chloride, as they present large and low uncertainties, respectively (see uncertainty values in Figure 6). Figure 7 presents the propagation of the uncertainty of $T_{\mathrm{c}}, p_{\mathrm{c}}, \omega$, and $c_{\mathrm{p}, 0}$ for 2,2-dichloropropane, by using the GCM and the ANN, through the PR-EoS for the calculation of the saturation pressure $p_{\text {sat }}$, saturated liquid and vapor densities (i.e., $\rho_{\text {liq }}, \rho_{\text {vap }}$ ), and saturated liquid and vapor enthalpies (i.e., $h_{\text {liq }}$, $\left.h_{\text {vap }}\right)$. Figure 8 presents the equivalent uncertainty propagation results for propyl chloride.
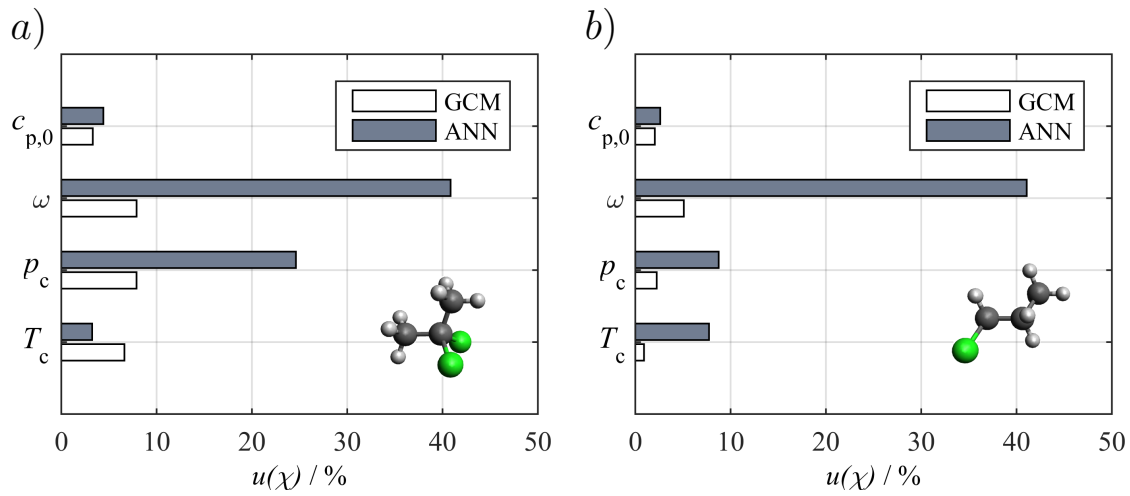

Figure 6: Estimated uncertainties of $T_{\mathrm{c}}, p_{\mathrm{c}}, \omega$, and $c_{\mathrm{p}, 0}$ (at $350 \mathrm{~K}$ ), for (a) 2,2dichloropropane and (b) propyl chloride, using both models. 
a)

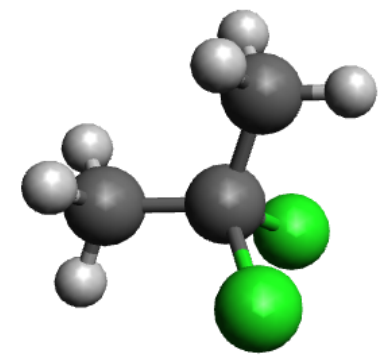

d)

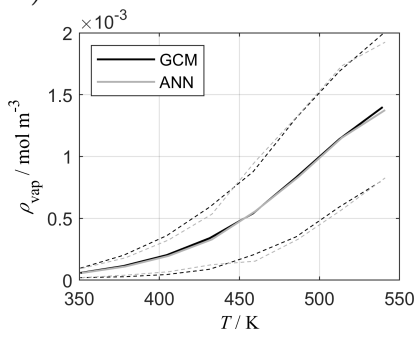

b)

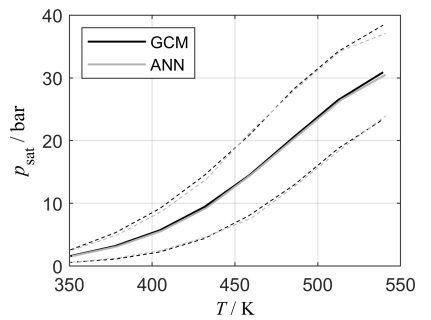

e)

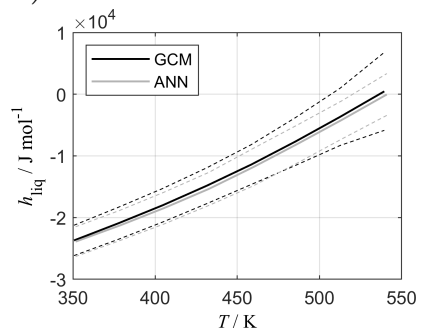

c)

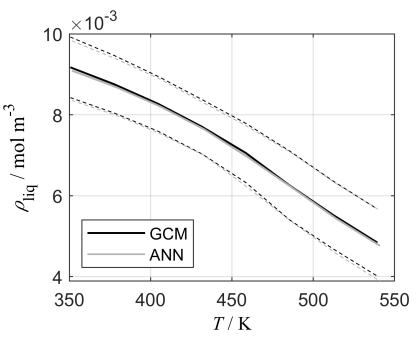

f)

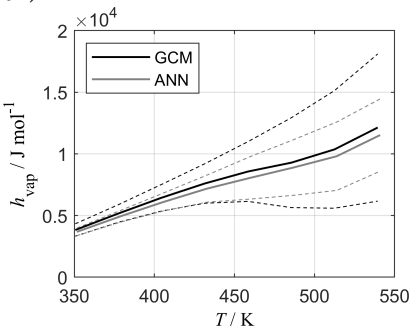

Figure 7: Example of propagation of the uncertainty of the estimated values of $T_{\mathrm{c}}, p_{\mathrm{c}}$, $\omega$, and $c_{\mathrm{p}, 0}$, for 2,2-dichloropropane (represented in (a) [30]) by using the GCM and the ANN, through the PR-EoS for the calculation of: (b) the saturation pressure $p_{\text {sat }},(\mathrm{c}, \mathrm{d})$ saturated liquid and vapor densities $\left(i . e ., \rho_{\text {liq }}, \rho_{\text {vap }}\right)$, and $(e, f)$ saturated liquid and vapor enthalpies (i.e., $\left.h_{\text {liq }}, h_{\mathrm{vap}}\right)$. Continuous lines represent the mean of the predicted property values for the 100 samples generated from the Monte Carlo simulation, while dashed lines represent their standard deviations. The predicted values of the critical temperature are: $T_{\mathrm{c}, \mathrm{GCM}}=539.46 \mathrm{~K}$ and $T_{\mathrm{c}, \mathrm{ANN}}=540.98 \mathrm{~K}$. 
a)

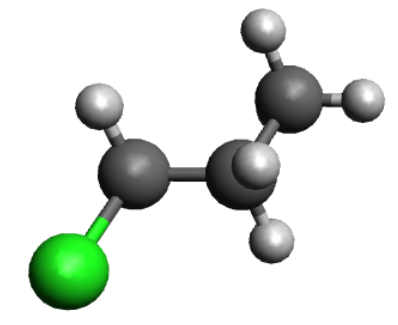

d)

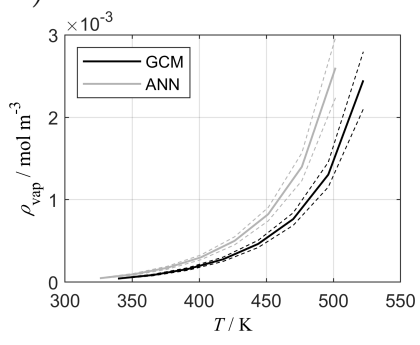

b)

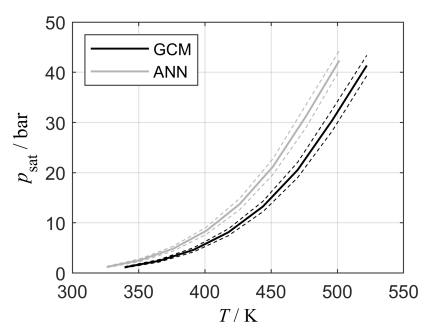

e)

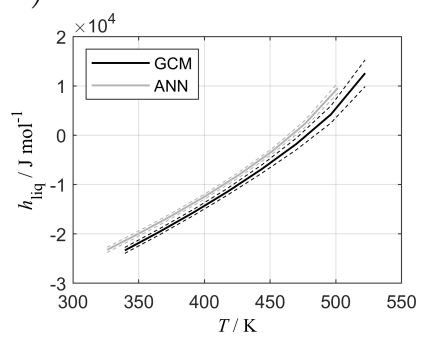

c)

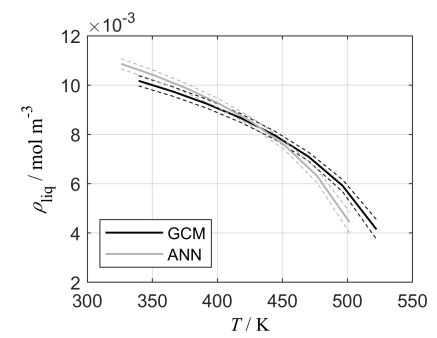

f)

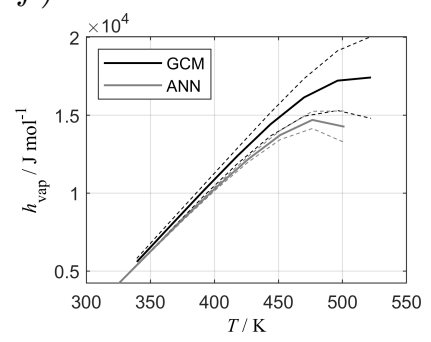

Figure 8: Example of propagation of the uncertainty of the estimated values of $T_{\mathrm{c}}, p_{\mathrm{c}}$ , $\omega$, and $c_{\mathrm{p}, 0}$, for propyl chloride (represented in (a) [30]) by using the GCM and the ANN, through the PR-EoS for the calculation of: (b) the saturation pressure $p_{\text {sat }},(\mathrm{c}, \mathrm{d})$ saturated liquid and vapor densities (i.e., $\left.\rho_{\text {liq }}, \rho_{\text {vap }}\right),(\mathrm{e}, \mathrm{f})$ and saturated liquid and vapor enthalpies (i.e., $\left.h_{\mathrm{liq}}, h_{\mathrm{vap}}\right)$. Continuous lines represent the mean of the predicted property values for the 100 samples generated from the Monte Carlo simulation, while dashed lines represent their standard deviations. The predicted values of the critical temperature are: $T_{\mathrm{c}, \mathrm{GCM}}=522.25 \mathrm{~K}$ and $T_{\mathrm{c}, \mathrm{ANN}}=501.54 \mathrm{~K}$.

As it can be observed, the uncertainty limits for both models are comparatively larger for 2,2-dichloropropane than for propyl chloride, being the uncertainties predicted by the ANN the greatest in most of the cases. The first fluid, with molecular formula $\mathrm{CH}_{3}-\mathrm{CCl}_{2}-\mathrm{CH}_{3}$, can be divided into the following first order groups (according to the groups in Ref. [4]): 2 groups $\mathrm{CH}_{3}$, and 1 group $\mathrm{CCl}_{2}$. As it can be seen in the correlation matrices represented in Figures 3 and 4 of the Supporting Information, the combination of the groups $\mathrm{CH}_{3}$ and $\mathrm{CCl}_{2}$ does not present a significantly strong correlation, however, the uncertainty associated with the functional group $\mathrm{CCl}_{2}$ for both the predicted $T_{\mathrm{c}}$ and $p_{\mathrm{c}}$ is high due to the fact that the group was present in only one fluid in the dataset. Propyl chloride, with formula $\mathrm{CH}_{2} \mathrm{Cl}-\mathrm{CH}_{2}-\mathrm{CH}_{3}$, can be divided into the following first order groups: 1 group $\mathrm{CH}_{3}, 1$ group $\mathrm{CH}_{2}$, and 1 group $\mathrm{CH}_{2} \mathrm{Cl}$. Although the correlation be- 
tween the two first groups is strong, due to the simultaneous presence of both groups in many molecules, the correlation with the group $\mathrm{CH}_{2} \mathrm{Cl}$ is low. This fact, together with the low uncertainties associated to the three groups for all properties (see Figure 2 in the Supporting Information), contribute to the low overall uncertainty of the properties for this molecule. It can be noted that there is no significant difference in magnitude for the saturated pressure and saturated densities between the GCM and the ANN. This is true even in the case of propyl chloride, which exhibits a significant difference in the uncertainty for the critical temperature, which was found to be one of the main contributors to the overall uncertainty. For this fluid, the main differences observed come from the difference in the predicted values of the critical temperature and pressure (about $20 \mathrm{~K}$ and 1 bar, respectively), which impact the calculated saturated pressures and densities. The propagated values of the uncertainties for the vapor pressure are in line with those observed by Hajipour and Satyro [7] in their study of the propagation of uncertainties on thermodynamic models. In the case of the saturated enthalpies, it can be seen that the uncertainty ranges increase at higher temperatures, and the uncertainty range is larger in the case of the saturated vapor enthalpy.

\subsection{Propagation of the uncertainty in a case study: organic Rankine cycle unit}

Figure 9 shows the values of the net power output and the thermal efficiency of the ORC unit when using R-1233zd(E) and R-1234yf as working fluids, calculated by using the predictions from both methods, with the estimated uncertainty indicated by the error bars, and Refprop [21]. Two interesting observations can be made. First, the estimated uncertainty for the net power output and thermal efficiency are of the same order of magnitude for both the GCM and the ANN for R-1233zd(E), although the ANN exhibits more uncertainty. However, the estimated value of the net power output by using the predicted properties by the ANN is closer to that obtained by using Refprop. This is due to the fact that, for this case, the GCM overestimates the $T_{\mathrm{c}}$ by almost $60 \mathrm{~K}$, which has a major impact on the calculation of the cycle pressures. For this case, the estimated uncertainties for the $T_{\mathrm{c}}$ are $8 \mathrm{~K}$ and $36 \mathrm{~K}$ for the $\mathrm{GCM}$ and the ANN, respectively. Also, the uncertainties for $p_{\mathrm{c}}$ and $\omega$ are larger for the ANN, which would explain the larger uncertainty in the net power output when using the values from the ANN. Second, in the case of R-1234yf, the results of both the net power output and efficiency for the GCM and the ANN differ substantially from 
those of Refprop. In this case, both models underestimate the $T_{\mathrm{c}}$ value by more than $10 \mathrm{~K}$, which has a major impact on the calculation of the cycle pressures and net power output. The overall uncertainty obtained for the ANN is high and can be a consequence of the high uncertainty associated with the group $\mathrm{CH}_{2}=\mathrm{C}$ of $\mathrm{R}-1234 \mathrm{yf}$, for the $p_{\mathrm{c}}$ model (see Figure 2 in the Supporting Information). Although the uncertainty in the predicted $T_{\mathrm{c}}$ by the ANN is of similar magnitude to that of $\mathrm{R}-1233 \mathrm{zd}(\mathrm{E})$, in this case it has a major impact on the calculation of the saturation pressures due to the slope of the saturation line of R-1234yf, thus increasing the uncertainty of the net power output. These cases may demonstrate that the uncertainty of the predicted values of a fluid's thermophysical properties will have a different impact on the prediction of net power output of an ORC unit, depending also on the deviation of their prediction and on the saturation line slope for that fluid.

a)

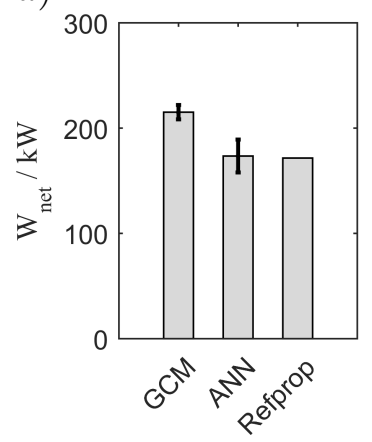

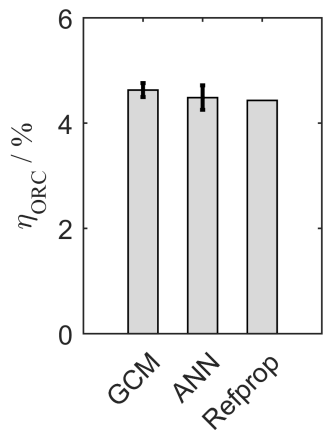

b)

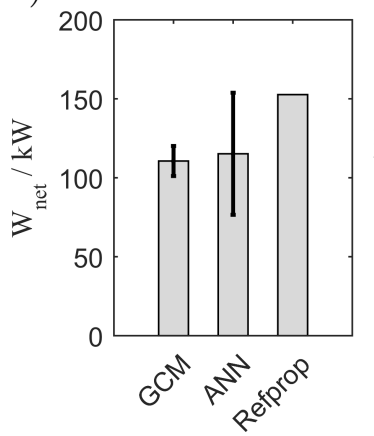

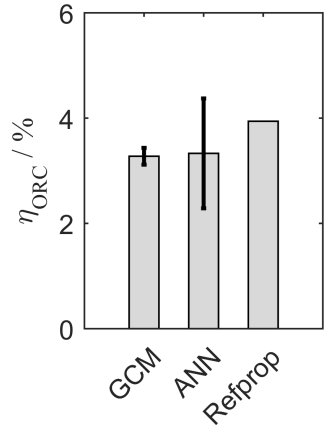

Figure 9: Comparison of the value and uncertainty of the prediction of $W_{\text {net }}$ and $\eta_{\text {ORC }}$ for a case using (a) R-1233zd(E) or (b) R-1234yf as the working fluid, for both the GCM and the ANN approaches. The values obtained by using Refprop are indicated as a reference.

\section{Discussion}

\subsection{Comparative analysis of the uncertainty of the GCM and the ANN}

As it was observed in section 3.1, no significant differences existed between the estimated uncertainty values of both models, except for the ideal gas heat capacity. Figure 10 depicts the average value of the calculated uncertainties for each method and property, and the standard deviation of the uncertainties. This figure indicates that the variance of the uncertainty obtained from the ANN is always lower than that of the GCM. This means 
that the uncertainty values obtained for the predictions of the GCM may be more sensitive to the functional groups forming the molecule, while the uncertainty of the ANN predictions will have similar values for different fluids. A possible reason for this can be that while the GCM parameters are more sensitive to the number of instances of the specific functional group in the fitting dataset, the ANN parameters are independent of this. This observation is also demonstrated in Figure 2 of the Supporting Information. This figure presents the uncertainty values obtained for $T_{\mathrm{c}}, p_{\mathrm{c}}, \omega$ and $c_{\mathrm{p}, 0}$ when only each of the functional groups is considered as an input, with the aim to search for correlations between molecular structure and overall uncertainty value. As it can be seen, while the variation in the ANN values is mostly negligible for all properties, the uncertainties of the GCM values vary to a larger extent for each functional group.
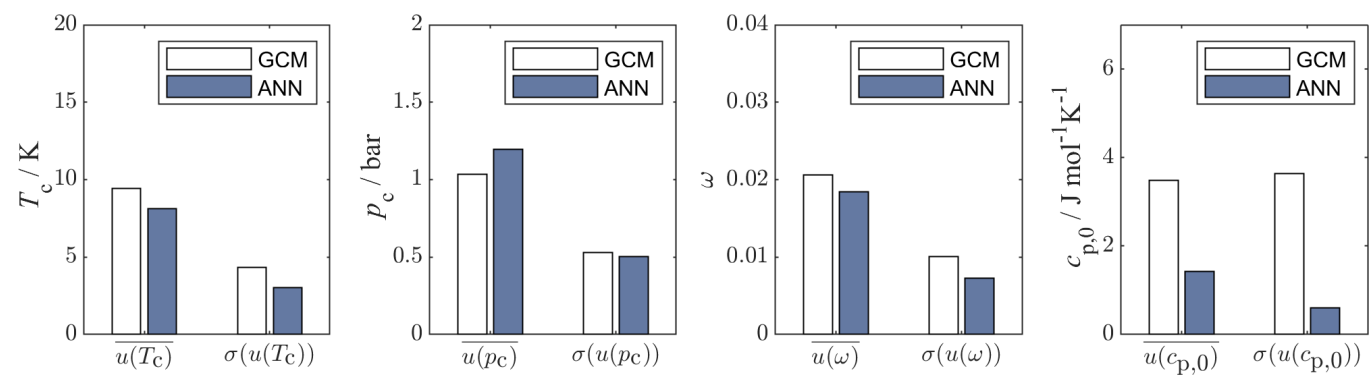

Figure 10: Average and standard deviation of the calculated uncertainties for the whole fluid dataset, for each of the considered properties (i.e., $T_{\mathrm{c}}, p_{\mathrm{c}}, \omega$, and $c_{\mathrm{p}, 0}$ ), and for each prediction model (GCM and ANN).

\subsection{Impact of the availability of experimental data on the propagated uncer- tainty}

Methods to predict thermophysical properties of fluids are of great utility in both industrial processes and research, but it is obvious that the high associated uncertainties of their calculated values can lead to inconclusive results when propagated in process simulations. Although the use of larger datasets for the fitting process of these methods can help reducing the uncertainty of the predictions, the uncertainty of the prediction cannot be less than the actual uncertainty of the available experimental data.

As mentioned in section 2.3.2, the uncertainty of the experimental data is not accounted for directly in the optimization of the group contribution or the neural network parameters. In some fitting procedures, not used here, the 
uncertainty of the experimental data is used only as a weight for the fitting process, but this still can result in estimated uncertainties less than those of the experimental data. Here, we compare the estimated uncertainties of the properties for those fluids for which data of their experimental uncertainties is available. Figure 11 shows this comparison by representing the estimated uncertainties of both GCM and ANN for the fluids in the dataset for which the experimental uncertainties of the critical temperature, critical pressure, acentric factor, and ideal gas heat capacity were available (i.e. selected data from the Project 801 of the Design Institute for Physical Property Data (DIPPR $\left.\left.{ }^{\circledR} 801\right)[23]\right)$.
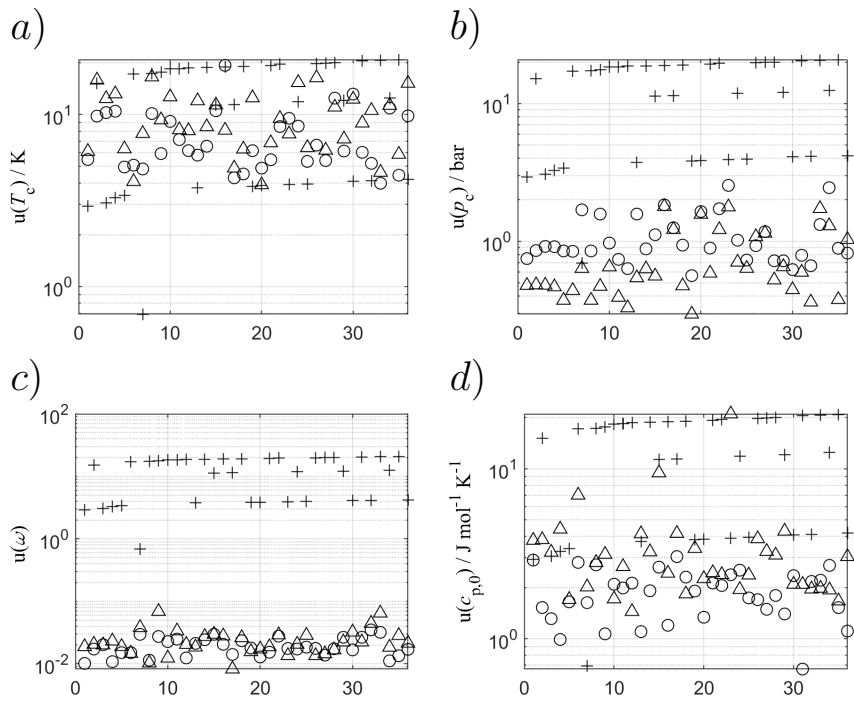

d)

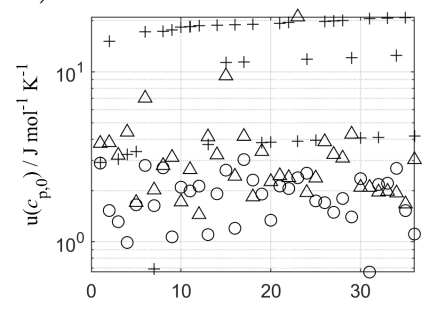

Figure 11: Comparison of the available experimental uncertainties with the predicted uncertainties from both models (GCM and ANN) for the same fluids. +: experimental uncertainty; $\triangle$ uncertainty obtained from the GCM; $\bigcirc$ uncertainty obtained from the ANN. Note the logarithmic scale of the y-axis.$$
\begin{aligned}
& \text { fitting process, due to their low number. Finally, for the acentric factor } \omega \text {, } \\
& \text { the experimental uncertainties are well below those of the models, indicating } \\
& \text { a more realistic situation. }
\end{aligned}
$$ 


\subsection{Comparison with other predictive methods}

Although comparing the deviation of different predictive models for specific inputs is easy, doing the equivalent comparison for the uncertainty of their predictions can be challenging. The main reason for this is that, as pointed out by Frutiger et al. [18], the uncertainty of the model predictions is rarely reported.

When it comes to the available predictive models for the properties and fluid groups treated in this work, this problem is also encountered. For instance, the models developed by Marrero and Gani [15], Constantinou et al. [31], and Joback and Reid [14] for the prediction of the critical temperature and critical pressure, acentric factor, and ideal gas heat capacity, respectively, do not provide information about the uncertainty of the predictions or the model parameters. Sobati et al. [32] provided the $95 \%$ confidence intervals of the parameters of their models based on the quantitative structure property relationship approach for the prediction of the critical temperature and critical pressure of pure refrigerants.

In order to illustrate how the uncertainties estimated in this work compare to the confidence intervals of the aforementioned model, Figure 12 presents the calculated values for 2,2-dichloropropane (circles) and 1,2-dichloro-1,1,2,3,3,3hexafluoro-propane (squares). For the considered fluids, the uncertainties for the critical pressure of the model of Sobati et al. [32] are similar to those of the GCM and the ANN, being slightly lower from the highest critical pressure value and slightly greater for the highest critical pressure value. In the case of the critical temperature, the results suggest that the uncertainties of the model of Sobati et al. [32] are greater than those of the ANN for both fluids, and they are only exceeded by the GCM for the highest critical temperature value. 
a)

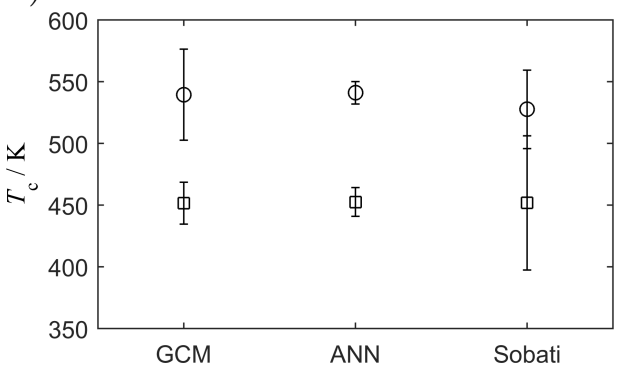

b)

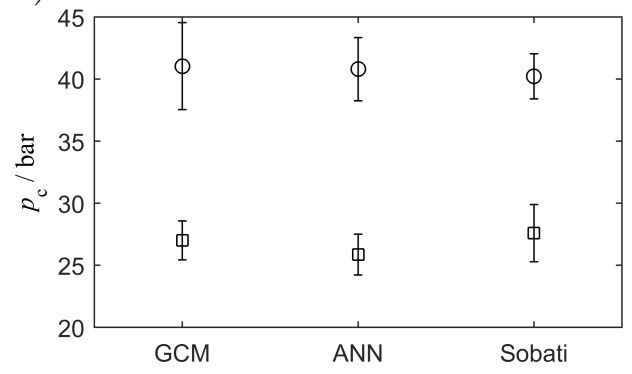

Figure 12: Estimated values and uncertainties of (a) the critical temperature $T_{\mathrm{c}}$ and (b) critical pressure $p_{\mathrm{c}}$ for 2,2-dichloropropane (circles) and 1,2-dichloro-1,1,2,3,3,3-hexafluoropropane (squares).

\subsection{Guidelines}

Based on the obtained results, a series of guidelines can be provided with regards to the expected uncertainty of new halogenated working fluids. In this section we summarize these findings in the form of guidelines with respect to the preferred method in order to reduce the uncertainty in the estimation of the properties depending on the molecular structure of the fluid.

As previously mentioned, the uncertainty values obtained from the GCM tend to vary more with the molecular structure of the fluid, while the uncertainty values obtained from the ANN are more homogeneous. This indicates that, in terms of the uncertainty of the prediction, the ANN value is more robust to changes in the molecular structure, which is an advantage in cases where the GCM yields high uncertainties in the predictions.

In this regard, Figure 2 in the Supporting Information provides a good insight on which groups are expected to add higher uncertainty to the prediction depending on the property and the model. A good way to predict for which fluids the predictions of the GCM can have higher uncertainties is by observing this Figure, which represents the uncertainty of each property when the specific functional group is used as input for the models. In the case of the critical temperature, molecules containing the functional groups $\mathrm{CH}=\mathrm{C}, \mathrm{CCl}$ or $\mathrm{CCl}_{2}$ are expected to yield larger uncertainties for the GCM. This can be motivated by the fact that these groups were underrepresented in the dataset used for the fitting of the GCM [4]. In the case of the critical pressure, only the functional group $\mathrm{CH}$ is expected to have greater uncertainty on the prediction from the GCM. For some of the groups, such as $\mathrm{CH}_{2}=\mathrm{C}$ or $\mathrm{CHF}_{2}$, the prediction of the GCM is expected to have a signifi- 
cantly lower uncertainty than that of the ANN. This can be motivated by a major presence of the mentioned group in the dataset used for the fitting of the models, or a lower degree of correlation of these groups with the rest of groups. In the case of the acentric factor, groups $\mathrm{CH}, \mathrm{CCl}$, and $\mathrm{CH}=\mathrm{C}$ are expected to yield larger uncertainties for the GCM. Finally, for the ideal gas heat capacity, it may be observed that several functional groups are expected to give a higher uncertainty for the GCM, especially in the case of HCClF.

Combining the predictive models for each property of a fluid depending on their expected relative deviations and uncertainty could help obtaining a prediction of its thermophysical behavior with low uncertainty.

\section{Supporting information}

The Supporting Information of this work contains the following material:

- An example of calculation of the uncertainty of the predicted values of $T_{\mathrm{c}}, p_{\mathrm{c}}, \omega$, and $c_{\mathrm{p}, 0}$.

- The uncertainty results for each of the individual groups for the models.

- The correlation matrices of the groups in the fluid set.

- The estimated uncertainties associated to the functional groups for the GCM.

The Matlab code of the function developed for the estimation of the uncertainty of the critical temperature, critical pressure, acentric factor, and ideal gas heat capacity of halogenated fluids using the GCM and the ANN is provided in the institutional repository (doi.org/10.11583/DTU.7438607), together with the needed variables of the predictive models. The repository link contains the following functions and variables:

- uncertainty model.m: obtains the estimated uncertainty for a specified model and property, as indicated in section 2.3.3. The input for its use is the array containing the number of groups for the fluid, except for the case of the ideal gas capacity, where the first element of the array will be the temperature in Kelvin. An example of use of this function can be found in Section 1 of the Supporting Information. 
- Tc model.mat: this is a structure that contains the array of experimental values of $T_{\mathrm{c}}$ (Tcexp), the fluid groups (groups), the models' parameters (param_GCM, param_ANN), the predictions of the GCM and the ANN (Tc_GCM, Tc_ANN), their uncertainties (u_GCM, u_ANN), and the PDF of both model parameters (pdf_GCM, pdf_ANN).

- Pc_model.mat: analogous to Tc_model.mat.

- w_model.mat: analogous to Tc_model.mat.

- cp0 model .mat: analogous to Tc model.mat.

- Tc_model.csv, Pc_model.csv, w_model.csv, and cp0_model.csv: contain the data of the .mat files in csv format.

\section{Conclusions}

In this work we estimated, analyzed, and compared the uncertainty of the predictions of the critical temperature, critical pressure, acentric factor, and ideal gas heat capacity of halogenated substances, by using two different predictive models. The propagation of these uncertainties through the use of the PR-EoS was studied, and a case study of an organic Rankine cycle unit was used to illustrate the effect of the uncertainties in process simulation. Based on the obtained results the following conclusions are drawn:

- On average, the estimated uncertainties of the critical temperature, acentric factor and the ideal gas heat capacity are lower for the model based on the ANN, while the uncertainties yielded by the GCM are lower for most of the considered fluids for the critical pressure.

- The estimated uncertainties from the GCM depend considerably on the molecular structure of the fluid, while the uncertainties yielded by the ANN predictions are less sensitive in this respect. As a consequence, the presence of some specific functional groups will add more uncertainty to the predicted value by using the GCM, and for fluids where those groups are present, the use of the ANN could be more recommendable.

- The GCM yields larger uncertainties on its predictions when the fluid molecule contains functional groups such as $\mathrm{CH}$ and $\mathrm{CCl}_{2}$. In those cases the ANN yields lower uncertainty values. 
- The uncertainty in the critical temperature is the main contributor to the overall uncertainty of properties calculated by using the PengRobinson equation of state, with a high influence on the saturated pressure and saturated liquid density and enthalpy.

- The propagation of the uncertainty of the critical parameters to the overall uncertainty of the net power output of an organic Rankine cycle unit is affected by different factors. Although the uncertainty of the critical temperature is the main contributor to the overall uncertainty, its combination with the uncertainty of the other parameters and the saturation behavior of the fluid can influence its impact on the total uncertainty.

- Although the uncertainty propagation in the prediction of the thermophysical properties of halogenated fluids has been exemplified on an ORC application, it must be noted that new halogenated substances are now being introduced as refrigerants in the automotive industry and in heat pumps. Therefore, the research presented in this work may be of interest, not only for the ORC technology, but also for the refrigeration industry.

\section{Acknowledgements}

The work documented in this paper has been funded by Den Danske Maritime Fond, project ID: 2015-070; DTU Mekanik, Organic Recycle Unit with the project Pilot-ORC (www. pilotORC.mek.dtu.dk), Innovationsfonden with the THERMCYC project (www.thermcyc.mek.dtu.dk, project ID: 130500036B) and the European Union's Horizon 2020 research and innovation programme with a Marie Skłodowska-Curie Fellowship under grant agreement No 704201 with the project NanoORC (www.nanoorc.mek.dtu.dk). The financial support is gratefully acknowledged. 


\section{References}

[1] Ozone Secretariat UNEP, Montreal protocol on substances that deplete the ozone layer, US Government Printing Office, 1987.

[2] Ozone Secretariat, United Nations Environment Programme, Synthesis of the reports of the Scientific, Environmental Effects, and Technology and Economic Assessment Panels of the Montreal Protocol1999.

[3] Regulation (EU) No 517/2014 of the European Parliament and of the Council of 16 April 2014 on fluorinated greenhouse gases and repealing, European Parliament, 2014.

[4] M. E. Mondejar, S. Cignitti, J. Abildskov, J. M. Woodley, F. Haglind, Prediction of properties of new halogenated olefins using two group contribution approaches, Fluid Phase Equilibria 433 (2017) 79-96.

[5] G. Sin, K. V. Gernaey, A. E. Lantz, Good modeling practice for pat applications: Propagation of input uncertainty and sensitivity analysis, Biotechnology Progress 25 (4) (2009) 1043-1053.

[6] W. B. Whiting, Effects of uncertainties in thermodynamic data and models on process calculations, Journal of Chemical \& Engineering Data 41 (5) (1996) 935-941.

[7] S. Hajipour, M. A. Satyro, Uncertainty analysis applied to thermodynamic models and process design-1. Pure components, Fluid Phase Equilibria 307 (1) (2011) 78-94.

[8] S. Hajipour, M. A. Satyro, M. W. Foley, Uncertainty analysis applied to thermodynamic models and process design-2. Binary mixtures, Fluid Phase Equilibria 364 (2014) 15-30.

[9] M. G. Bjørner, G. Sin, G. M. Kontogeorgis, Uncertainty analysis of the CPA and a quadrupolar CPA equation of state-With emphasis on CO2, Fluid Phase Equilibria 414 (2016) 29-47.

[10] M. Shacham, N. Braunerb, M. B. Cutlipc, Considering physical property uncertainties in process design, in: Symposium on Computer Aided Process Engineering, Vol. 17, 2012, p. 20. 
[11] A. Hukkerikar, M. N. Jones, B. Sarup, J. Abildskov, G. Sin, R. Gani, Sensitivity of Process Design due to Uncertainties in Property Estimates, in: 11th International Symposium on Process Systems EngineeringPSE2012, Vol. 15, Elsevier, 2012, p. 200.

[12] J. Frutiger, I. Bell, J. P. O'Connell, K. Kroenlein, J. Abildskov, G. Sin, Uncertainty assessment of equations of state with application to an organic Rankine cycle, Molecular Physics (2017) 1-20.

[13] J. Frutiger, J. Andreasen, W. Liu, H. Spliethoff, F. Haglind, J. Abildskov, G. Sin, Working fluid selection for organic Rankine cycles-Impact of uncertainty of fluid properties, Energy 109 (2016) 987-997.

[14] K. G. Joback, R. C. Reid, Estimation of pure-component properties from group-contributions, Chemical Engineering Communications 57 (16) (1987) 233-243.

[15] J. Marrero, R. Gani, Group-contribution based estimation of pure component properties, Fluid Phase Equilibria 183 (2001) 183-208.

[16] A. S. Hukkerikar, B. Sarup, A. Ten Kate, J. Abildskov, G. Sin, R. Gani, Group-contribution $+(\mathrm{gc}+)$ based estimation of properties of pure components: Improved property estimation and uncertainty analysis, Fluid Phase Equilibria 321 (2012) 25-43.

[17] R. Gani, P. M. Harper, M. Hostrup, Automatic creation of missing groups through connectivity index for pure-component property prediction, Industrial \& Engineering Chemistry Research 44 (18) (2005) $7262-7269$.

[18] J. Frutiger, C. Marcarie, J. Abildskov, G. Sin, A Comprehensive Methodology for Development, Parameter Estimation, and Uncertainty Analysis of Group Contribution Based Property Models: An Application to the Heat of Combustion, Journal of Chemical \& Engineering Data 61 (1) (2015) 602-613.

[19] M. L. Michelsen, J. Mollerup, Thermodynamic Modelling: Fundamentals and Computational Aspects, Tie-Line Publications, 2004. 
[20] J. S. Brown, Predicting performance of refrigerants using the PengRobinson Equation of State, International Journal of Refrigeration 30 (8) (2007) 1319-1328.

[21] E. Lemmon, M. Huber, M. McLinden, NIST Standard Reference Database 23: Reference Fluid Thermodynamic and Transport Properties-REFPROP, Version 9.1 (2013).

[22] W. Liu, D. Meinel, C. Wieland, H. Spliethoff, Investigation of hydrofluoroolefins as potential working fluids in organic Rankine cycle for geothermal power generation, Energy 67 (2014) 106-116.

[23] R. Rowley, W. Wilding, L. Oscarson, T. Knotts, N. Giles, DIPPR@ Data Compilation of Pure Chemical Properties, AIChE, New York, 2014.

[24] F. A. Aly, L. L. Lee, Self-consistent equations for calculating the ideal gas heat capacity, enthalpy, and entropy, Fluid Phase Equilibria 6 (3) (1981) 169-179.

[25] R. Feistel, J. Lovell-Smith, P. Saunders, S. Seitz, Uncertainty of empirical correlation equations, Metrologia 53 (4) (2016) 1079.

[26] R. H. Lopes, Kolmogorov-smirnov test, in: International Encyclopedia of Statistical Science, Springer, 2011, pp. 718-720.

[27] J. C. Helton, F. J. Davis, Latin hypercube sampling and the propagation of uncertainty in analyses of complex systems, Reliability Engineering \& System Safety 81 (1) (2003) 23-69.

[28] R. L. Iman, W.-J. Conover, A distribution-free approach to inducing rank correlation among input variables, Communications in StatisticsSimulation and Computation 11 (3) (1982) 311-334.

[29] J. G. Andreasen, A. Meroni, F. Haglind, A Comparison of Organic and Steam Rankine Cycle Power Systems for Waste Heat Recovery on Large Ships, Energies 10 (4) (2017) 547.

[30] M. D. Hanwell, D. E. Curtis, D. C. Lonie, T. Vandermeersch, E. Zurek, G. R. Hutchison, Avogadro: an advanced semantic chemical editor, visualization, and analysis platform, Journal of Cheminformatics 4 (1) (2012) 17. 
827

828

829

830

831

832

[31] L. Constantinou, R. Gani, J. P. O'Connell, Estimation of the acentric factor and the liquid molar volume at $298 \mathrm{~K}$ using a new group contribution method, Fluid Phase Equilibria 103 (1) (1995) 11-22.

[32] M. A. Sobati, D. Abooali, Molecular based models for estimation of critical properties of pure refrigerants: Quantitative structure property relationship (QSPR) approach, Thermochimica Acta 602 (2015) 53-62. 